\title{
A NOTE ON BOGOMOLOV-GIESEKER TYPE INEQUALITY FOR CALABI-YAU 3-FOLDS
}

\author{
YUKINOBU TODA \\ (Communicated by Lev Borisov)
}

\begin{abstract}
The conjectural Bogomolov-Gieseker (BG) type inequality for tilt semistable objects on projective 3-folds was proposed by Bayer, Macri and the author. In this note, we prove our conjecture for slope stable sheaves with the smallest first Chern class on certain Calabi-Yau 3-folds, e.g. quintic 3-folds.
\end{abstract}

\section{INTRODUCTION}

1.1. Motivation and result. Let $X$ be a smooth projective 3 -fold over $\mathbb{C}$. Given an element

$$
B+i \omega \in H^{2}(X, \mathbb{C})
$$

with $\omega$ ample, the heart of a bounded t-structure

$$
\mathcal{B}_{B, \omega} \subset D^{b} \operatorname{Coh}(X)
$$

was constructed in [3] following the construction of Bridgeland's stability conditions on projective surfaces [5, [1. The notion of tilt stability on $\mathcal{B}_{B, \omega}$ was introduced in [3], and a conjectural Bogomolov-Gieseker (BG) type inequality among Chern characters of tilt semistable objects in $\mathcal{B}_{B, \omega}$ was proposed in [3, Conjecture 1.3.1]. Our conjecture in [3] turned out to imply several very important results: construction of Bridgeland stability on projective 3-folds [3, Fujita's conjecture in birational geometry [2], and Ooguri-Strominger-Vafa's conjecture in string theory [9]. In this note, we report partial progress toward the conjectural BG type inequality in 3 .

When $B=0$, the first Chern class on the heart $\mathcal{B}_{0, \omega}$ is always non-negative and plays a role in the rank on the category of coherent sheaves. A hopeful approach toward the proof of the main conjecture in [3] is to use the induction argument on the first Chern classes of tilt semistable objects, as in the proof of BG type inequality without $\mathrm{ch}_{3}$ (cf. [3, Theorem 7.3]). As a first step for this induction argument, the conjectural BG type inequality should be solved when the tilt semistable object has the smallest first Chern class. In this case, the main conjecture in [3] is formulated to be the following conjecture for slope stable sheaves (cf. [3, Conjecture 7.2.3]) 1$]$

Received by the editors January 29, 2012 and, in revised form, October 30, 2012.

2010 Mathematics Subject Classification. Primary 14F05.

This work was supported by a World Premier International Research Center Initiative (WPI initiative), MEXT, Japan. This work was also supported by Grant-in-Aid for Scientific Research grant (22684002), and partly (S-19104002), from the Ministry of Education, Culture, Sports, Science and Technology, Japan.

${ }^{1}$ The statement of [3] Conjecture 7.2.3] was more general than Conjecture 1.1 and the formulation is slightly different. When $\operatorname{Pic}(X)$ is generated by one element, they are obviously equivalent. 
Conjecture 1.1. Let $X$ be a smooth projective 3 -fold such that $\operatorname{Pic}(X)$ is generated by $\mathcal{O}_{X}(H)$ for an ample divisor $H$ in $X$. Then for any torsion free slope stable sheaf $E$ on $X$ with $c_{1}(E)=[H]$ and $\operatorname{ch}_{2}(E) H>0$, we have the inequality,

$$
\operatorname{ch}_{3}(E) \leq \frac{\operatorname{ch}_{2}(E) H}{3 \operatorname{ch}_{0}(E)} .
$$

The above conjecture was studied in [3, Example 7.2.4] for rank one torsion free sheaves. In this case, inequality (10) is reduced to Castelnuovo type inequality for low degree curves in $X$. On the other hand, the higher rank case was not studied in [3]. The purpose of this article is to show that, when $X$ is a certain CalabiYau 3-fold, the inequality (1) is reduced to Castelnuovo type inequality even in the higher rank case. The main result is as follows:

Theorem 1.2. Let $X$ be a smooth projective Calabi-Yau 3-fold such that $\operatorname{Pic}(X)$ is generated by $\mathcal{O}_{X}(H)$ for an ample divisor $H$ in $X$. Suppose that the following inequalities hold:

$$
\begin{aligned}
\operatorname{dim}|H| & \geq \frac{7}{6} H^{3}-3, \\
\chi\left(\mathcal{O}_{C}\right) & \geq \frac{1}{6} H^{3}-C \cdot H,
\end{aligned}
$$

for any one dimensional subscheme $C \subset X$ with $C \cdot H<H^{3} / 2$. Then $X$ satisfies Conjecture 1.1. Furthermore inequality (11) is an equality only when $E=\mathcal{O}_{X}(H)$.

As we discussed in [3. Example 7.2.4], a typical (and important) example satisfying the assumption of Theorem 1.2 is a quintic 3 -fold in $\mathbb{P}^{4}$. Therefore we obtain the following corollary:

Corollary 1.3. Let $X \subset \mathbb{P}^{4}$ be a smooth quintic 3 -fold. Then $X$ satisfies Conjecture 1.1 .

In the case of quintic 3 -folds, the conditions $c_{1}(E)=[H], \operatorname{ch}_{2}(E) H>0$ and the Bogomolov-Gieseker inequality [4, 6] restrict the rank of $E$ up to five. So a priori, the sheaf $E$ could be $\operatorname{rank}(E) \geq 2$. On the other hand, we do not know of any example of such a sheaf $E$ with $\operatorname{rank}(E) \geq 2$ (cf. Remark 2.5). The result of Corollary 1.3 means that $\operatorname{ch}_{3}(E)$ should obey the desired inequality (1), if such a sheaf $E$ exists.

In general, the third Chern character $\operatorname{ch}_{3}(E)$ is known to be bounded by a certain polynomial of $\operatorname{ch}_{0}(E), \operatorname{ch}_{1}(E)$ and $\operatorname{ch}_{2}(E)$; see [8]. However, the evaluation in 8] is not strict to show inequality (1). Although the hypersurface restriction of $E$ plays an important role in [8], we do not take the hypersurface restriction. Instead we take the universal extension and the classical Bogomolov-Gieseker inequality to evaluate the dimensions of cohomology groups. As far as the author knows, such a method is not seen in literature.

1.2. Notation and convention. In this note, all the varieties are defined over $\mathbb{C}$. For $E, F \in \operatorname{Coh}(X)$, we denote $h^{i}(E):=\operatorname{dim} H^{i}(X, E), \operatorname{ext}^{i}(E, F):=\operatorname{dim}_{\operatorname{Ext}^{i}}(E, F)$ and $r(E):=\operatorname{rank}(E)$. We say $X$ is a Calabi-Yau 3 -fold if $\operatorname{dim} X=3$, its canonical line bundle is trivial and $h^{1}\left(\mathcal{O}_{X}\right)=0$. For an ample divisor $H$ in a 3-fold $X$ and a torsion free sheaf $E$ on $X$, its slope is denoted by

$$
\mu_{H}(E):=\frac{c_{1}(E) H^{2}}{r(E)} .
$$


The notion of slope stability is defined in the usual way (cf. [7]). For a subscheme $Z \subset X$, the defining ideal sheaf of $Z$ is denoted by $I_{Z}$.

\section{Proof of Theorem 1.2}

2.1. Some lemmas. The key ingredient for the proof of Theorem 1.2 is the following two lemmas, which may be well-known. For instance, there is a result in [10. Lemma 1.1] for K3 surfaces similar to Lemma 2.1. For the reader's convenience, we give the proofs.

Lemma 2.1. Let $X$ be a smooth projective Calabi-Yau 3-fold such that $\operatorname{Pic}(X)$ is generated by $\mathcal{O}_{X}(H)$ for an ample divisor $H$ in $X$. Let $E$ be a torsion free slope stable sheaf with $c_{1}(E)=[H]$, and set $V:=\operatorname{Ext}^{1}\left(E, \mathcal{O}_{X}\right)^{\vee}$. Then if we take the universal extension

$$
0 \rightarrow V \otimes_{\mathbb{C}} \mathcal{O}_{X} \rightarrow E^{\prime} \rightarrow E \rightarrow 0
$$

the sheaf $E^{\prime}$ is also slope stable.

Proof. We prove the assertion by induction on $\operatorname{ext}^{1}\left(E, \mathcal{O}_{X}\right)$. When $\operatorname{ext}^{1}\left(E, \mathcal{O}_{X}\right)=$ 0 , then the assertion is obvious.

Suppose that $\operatorname{ext}^{1}\left(E, \mathcal{O}_{X}\right)>0$, and take a non-zero element $a \in \operatorname{Ext}^{1}\left(E, \mathcal{O}_{X}\right)$. We have the extension

$$
0 \rightarrow\langle a\rangle^{\vee} \otimes_{\mathbb{C}} \mathcal{O}_{X} \rightarrow E_{a} \rightarrow E \rightarrow 0
$$

where $\langle a\rangle$ is the one dimensional subspace of $\operatorname{Ext}^{1}\left(E, \mathcal{O}_{X}\right)$ spanned by $a$. We show that $E_{a}$ is slope stable. Suppose by contradiction that $E_{a}$ is not slope stable. Then there is a saturated subsheaf $F \subset E_{a}$ such that $F$ is slope stable and

$$
\mu_{H}(F) \geq \mu_{H}\left(E_{a}\right), \quad r(F)<r\left(E_{a}\right)=r(E)+1 .
$$

If we write $c_{1}(F)=k[H]$, then $k \geq 1$; hence $\operatorname{Hom}\left(F, \mathcal{O}_{X}\right)=0$. It follows that the composition

$$
F \subset E_{a} \rightarrow E
$$

is non-zero, which implies $\mu_{H}(F) \leq \mu_{H}(E)$. Combined with (6), we obtain the inequality

$$
\frac{H^{3}}{r(E)+1} \leq \frac{k H^{3}}{r(F)} \leq \frac{H^{3}}{r(E)}
$$

The above inequality immediately implies $k=1$ and $r(F)=r(E)$. Then $\mu_{H}(F)=$ $\mu_{H}(E)$, and since $F$ and $E$ are slope stable with the same slope, the non-zero morphism (7) is an isomorphism. However this contradicts the fact that the sequence (5) is non-split.

Let $V_{a}$ be the $\mathbb{C}$-vector space $\operatorname{Ext}^{1}\left(E_{a}, \mathcal{O}_{X}\right)^{\vee}$ and take the universal extension,

$$
0 \rightarrow V_{a} \otimes_{\mathbb{C}} \mathcal{O}_{X} \rightarrow E_{a}^{\prime} \rightarrow E_{a} \rightarrow 0 .
$$

Applying $\operatorname{Hom}\left(-, \mathcal{O}_{X}\right)$ to the sequence (5), we obtain the exact sequence

$$
0 \rightarrow V_{a} \rightarrow V \rightarrow\langle a\rangle^{\vee} \rightarrow 0 .
$$

Hence $E_{a}^{\prime}$ is slope stable by the assumption of the induction. It is enough to show that $E^{\prime} \cong E_{a}^{\prime}$. 
By the construction of the exact sequences (4), (5), we have the commutative diagram

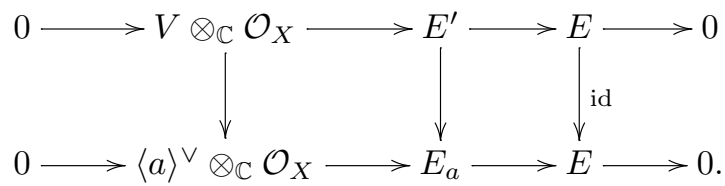

By the snake lemma and (9), we obtain the exact sequence

$$
0 \rightarrow V_{a} \otimes_{\mathbb{C}} \mathcal{O}_{X} \rightarrow E^{\prime} \rightarrow E_{a} \rightarrow 0 .
$$

The above sequence is isomorphic to the sequence (8). Indeed, by applying $\operatorname{Hom}\left(*, \mathcal{O}_{X}\right)$ to the sequence (44), it follows that $\operatorname{Ext}^{1}\left(E^{\prime}, \mathcal{O}_{X}\right)=0$ by the universality of (4). This in turn implies that the sequence (10) induces an isomorphism $V_{a}^{\vee} \cong \operatorname{Ext}^{1}\left(E_{a}, \mathcal{O}_{X}\right)$. Hence (10) is also the universal extension and is isomorphic to (8). Therefore $E^{\prime} \cong E_{a}^{\prime}$ follows.

Lemma 2.2. In the situation of Lemma 2.1, suppose that $r(E) \geq 2$ and there is a non-zero element $s \in H^{0}(X, E)$. Then for the associated exact sequence

$$
0 \rightarrow \mathcal{O}_{X} \stackrel{s}{\rightarrow} E \rightarrow F \rightarrow 0
$$

the sheaf $F$ is also slope stable.

Proof. We first show that $F$ is torsion free. If $F$ has a torsion, there is an exact sequence

$$
0 \rightarrow \mathcal{O}_{X} \rightarrow A \rightarrow T \rightarrow 0
$$

where $T$ is a non-zero torsion sheaf and $A \subset E$ is a rank one torsion free sheaf. If $\operatorname{dim} \operatorname{Supp}(T)=2$, then $c_{1}(A)=k[H]$ with $k \geq 1$, which contradicts the fact that $E$ is slope stable. Therefore $\operatorname{dim} \operatorname{Supp}(T) \leq 1$, and hence

$$
\begin{aligned}
\operatorname{Ext}^{1}\left(T, \mathcal{O}_{X}\right) & \cong H^{2}(X, T)^{\vee} \\
& \cong 0 .
\end{aligned}
$$

Therefore the sequence (11) splits, which contradicts the fact that $A$ is torsion free.

Next suppose that $F$ is not slope stable. Then there is a slope stable sheaf $G$ and a surjection $F \rightarrow G$ satisfying

$$
\mu_{H}(G) \leq \mu_{H}(F), \quad r(G)<r(F)=r(E)-1 .
$$

Also since there is a surjection $E \rightarrow F \rightarrow G$ and $E, G$ are slope stable, we have $\mu_{H}(E) \leq \mu_{H}(G)$. Hence if we write $c_{1}(G)=k[H]$, we obtain the inequality

$$
\frac{H^{3}}{r(E)} \leq \frac{k H^{3}}{r(G)} \leq \frac{H^{3}}{r(E)-1}
$$

It is immediate to see that there is no solution $(k, r(G))$ satisfying the above inequality and $r(G)<r(E)-1$. Hence $F$ is slope stable.

As a corollary of Lemma 2.2, we have the following:

Corollary 2.3. In the situation of Lemma 2.1, there is an exact sequence of the form

$$
0 \rightarrow \mathcal{O}_{X}^{\oplus m} \rightarrow E \rightarrow F \rightarrow 0
$$


such that $F$ is either a rank one torsion free sheaf or a slope stable sheaf with $r(F) \geq 2$ and $h^{0}(F)=0$.

Proof. We show the assertion by induction on $\theta(E)$, which is defined by

$$
\theta(E):=\min \left\{h^{0}(E), r(E)-1\right\} .
$$

The assertion is obvious when $\theta(E)=0$. Suppose that $\theta(E)>0$, i.e. $h^{0}(E) \neq 0$ and $r(E) \geq 2$. Then there is a non-zero element $s \in H^{0}(X, E)$. If we take the exact sequence

$$
0 \rightarrow \mathcal{O}_{X} \stackrel{s}{\rightarrow} E \rightarrow F_{s} \rightarrow 0,
$$

then $F_{s}$ is slope stable by Lemma 2.2, By applying $\operatorname{Hom}\left(\mathcal{O}_{X},-\right)$ to the sequence (13), we see $h^{0}\left(F_{s}\right)=h^{0}(E)-1$. Hence we have $\theta\left(F_{s}\right)=\theta(E)-1$, and by the assumption of the induction there is an exact sequence

$$
0 \rightarrow \mathcal{O}_{X}^{\oplus m^{\prime}} \rightarrow F_{s} \rightarrow F \rightarrow 0
$$

such that $F$ is a rank one torsion free sheaf or a slope stable sheaf with $r(F) \geq 2$ and $h^{0}(F)=0$. The desired exact sequence (12) is obtained by combining sequence (14) with (13).

\subsection{Proof of Theorem 1.2.}

Proof. Let $X$ be as in the statement of Theorem 1.2, and $E$ a slope stable sheaf on $X$ with $c_{1}(E)=[H]$ and $\operatorname{ch}_{2}(E) H>0$. By Corollary 2.3, there is an exact sequence of the form

$$
0 \rightarrow \mathcal{O}_{X}^{\oplus m} \rightarrow E \rightarrow F \rightarrow 0
$$

such that either $F$ is a rank one torsion free sheaf or a slope stable sheaf with $r(F) \geq 2$ and $h^{0}(F)=0$. Note that in the first case we have $F \cong \mathcal{O}_{X}(H) \otimes I_{Z}$ for a subscheme $Z \subset X$ with $\operatorname{dim} Z \leq 1$. We evaluate $\operatorname{ch}_{3}(E)=\operatorname{ch}_{3}(F)$ by dividing into the following three cases:

Case 1 . We have $F \cong \mathcal{O}_{X}(H) \otimes I_{Z}$ with $\operatorname{dim} Z=0$.

In this case, we have

$$
\operatorname{Ext}^{1}\left(F, \mathcal{O}_{X}\right) \cong H^{2}(X, F)^{\vee}
$$

by the Serre duality, which is zero by the cohomology exact sequence associated to the sequence

$$
0 \rightarrow \mathcal{O}_{X}(H) \otimes I_{Z} \rightarrow \mathcal{O}_{X}(H) \rightarrow \mathcal{O}_{Z} \rightarrow 0
$$

and the Kodaira vanishing $h^{2}\left(\mathcal{O}_{X}(H)\right)=0$. Hence sequence (15) splits if $m>0$, which contradicts the slope stability of $E$. Therefore $E \cong \mathcal{O}_{X}(H) \otimes I_{Z}$ and

$$
\begin{aligned}
\operatorname{ch}_{3}(E) & =\frac{1}{6} H^{3}-\text { length } \mathcal{O}_{Z}, \\
\frac{\operatorname{ch}_{2}(E)}{3 \operatorname{ch}_{0}(E)} & =\frac{1}{6} H^{3}-\frac{1}{3} \text { length } \mathcal{O}_{Z} .
\end{aligned}
$$

The above equalities imply inequality (11), and the equality holds only when $Z=\emptyset$.

Case 2. We have $F \cong \mathcal{O}_{X}(H) \otimes I_{Z}$ with $\operatorname{dim} Z=1$. 
In this case, $\operatorname{ch}_{2}(E) H=\operatorname{ch}_{2}(F) H>0$ is equivalent to

$$
0<H \cdot Z<\frac{1}{2} H^{3} .
$$

Applying assumption (3), we have

$$
\begin{aligned}
\operatorname{ch}_{3}(E) & =\operatorname{ch}_{3}(F) \\
& =\frac{1}{6} H^{3}-H \cdot[Z]-\chi\left(\mathcal{O}_{Z}\right) \\
& \leq 0 .
\end{aligned}
$$

Therefore inequality (1) holds.

Case 3. We have $r(F) \geq 2$ and $h^{0}(F)=0$.

By the Riemann-Roch theorem, we have

$$
\begin{aligned}
\chi(E) & :=\sum_{i \geq 0}(-1)^{i} h^{i}(E) \\
& =\operatorname{ch}_{3}(E)+\frac{1}{12} H \cdot c_{2}(X) \\
& =\operatorname{ch}_{3}(E)+\operatorname{dim}|H|-\frac{1}{6} H^{3}+1 .
\end{aligned}
$$

Here the last equality follows from the Riemann-Roch theorem applied for $\mathcal{O}_{X}(H)$ and the Kodaira vanishing $h^{i}\left(\mathcal{O}_{X}(H)\right)=0$ for $i>0$. On the other hand, since $\chi\left(\mathcal{O}_{X}\right)=0$ and $h^{0}(F)=0$, we have

$$
\chi(E)=\chi(F) \leq h^{2}(F)=\operatorname{ext}^{1}\left(F, \mathcal{O}_{X}\right) .
$$

Here the last equality follows from the Serre duality. Let us take the universal extension

$$
0 \rightarrow \mathcal{O}_{X}^{\oplus \operatorname{ext}^{1}\left(F, \mathcal{O}_{X}\right)} \rightarrow F^{\prime} \rightarrow F \rightarrow 0 .
$$

By Lemma 2.1, the sheaf $F^{\prime}$ is slope stable satisfying

$$
\begin{aligned}
\operatorname{ch}_{0}\left(F^{\prime}\right) & =\operatorname{ch}_{0}(F)+\operatorname{ext}^{1}\left(F, \mathcal{O}_{X}\right), \\
\operatorname{ch}_{i}\left(F^{\prime}\right) & =\operatorname{ch}_{i}(F)=\operatorname{ch}_{i}(E), i \geq 1 .
\end{aligned}
$$

By the Bogomolov-Gieseker inequality [4, [6] applied for $F^{\prime}$, we obtain the inequality

$$
\left(H^{2}-2\left(\operatorname{ch}_{0}(F)+\operatorname{ext}^{1}\left(F, \mathcal{O}_{X}\right)\right) \operatorname{ch}_{2}(E)\right) H \geq 0 .
$$

Since $\operatorname{ch}_{2}(E) H>0$ by the assumption, the above inequality is equivalent to

$$
\operatorname{ext}^{1}\left(F, \mathcal{O}_{X}\right) \leq \frac{H^{3}}{2 \operatorname{ch}_{2}(E) H}-\operatorname{ch}_{0}(F) \text {. }
$$

By (16), (17), (18) and noting $\operatorname{ch}_{2}(E) H \geq 1 / 2, \operatorname{ch}_{0}(F) \geq 2$, we obtain the inequality

$$
\begin{aligned}
\operatorname{ch}_{3}(E) & \leq \frac{H^{3}}{2 \operatorname{ch}_{2}(E) H}-\operatorname{ch}_{0}(F)+\frac{1}{6} H^{3}-\operatorname{dim}|H|-1 \\
& \leq \frac{7}{6} H^{3}-\operatorname{dim}|H|-3 \\
& \leq 0 .
\end{aligned}
$$

The last inequality follows from assumption (2). Therefore inequality (1) holds. 
Remark 2.4. When $H^{3}$ is even, we have $\operatorname{ch}_{2}(E) H \geq 1$; hence we have the better inequality in (19). Consequently, we can weaken assumption (2) to be

$$
\operatorname{dim}|H| \geq \frac{2}{3} H^{3}-3
$$

For instance, the complete intersections

$$
(2,4) \subset \mathbb{P}^{5},(2,2,3) \subset \mathbb{P}^{6}
$$

satisfy the above inequality. However the Castelnuovo type inequality (3) remains open, as we discussed in [3, Example 7.2.4].

Remark 2.5. Let $X$ be a quintic 3-fold and $E$ a slope stable sheaf $E$ on $X$ satisfying the assumption of Conjecture 1.1. Then the Bogomolov-Gieseker inequality restricts the possibility for the vector $\left(r(E), c_{2}(E) H\right)$ to be one of the following:

$$
(1,0),(1,1),(1,2),(2,2),(3,2),(4,2),(5,2) \text {. }
$$

However we don't know any example of $E$ with $r(E) \geq 2$ and $c_{2}(E) H=2$.

Remark 2.6. The result of Corollary 1.3 easily shows that the conjecture in 3 , Conjecture 1.3.1] is true if $X$ is a quintic 3-fold, $B=0$ and $c_{1}(E)=[H]$.

Remark 2.7. When $X$ is a quintic 3-fold, the proof of Theorem 1.2 shows that $\operatorname{ch}_{3}(E) \leq 0$, except $E=\mathcal{O}_{X}(H)$.

Remark 2.8. The exact sequences of sheaves (41), (12) are nothing but exact sequences

$$
\begin{aligned}
& 0 \rightarrow E^{\prime} \rightarrow E \rightarrow V \otimes_{\mathbb{C}} \mathcal{O}_{X}[1] \rightarrow 0, \\
& 0 \rightarrow E \rightarrow F \rightarrow \mathcal{O}_{X}^{\oplus m}[1] \rightarrow 0,
\end{aligned}
$$

in the tilted heart $\mathcal{B}_{0, H} \subset D^{b} \operatorname{Coh}(X)$. This observation may be helpful for the study of [3, Conjecture 1.3.1] when the first Chern class of $E$ is higher than $[H]$.

Remark 2.9. The result of Corollary 1.3 also implies a partial result on [3, Conjecture 1.3.1] when $X$ is a quintic 3-fold, $B=0$ and $c_{1}(E)=2[H]$. Indeed, in the notation of [3], suppose that $E \in \mathcal{B}_{0, H}$ satisfies $c_{1}(E)=2[H]$ and $\nu_{t[H]}(E)=0$ for some $t \in \mathbb{R}_{>0}$. If $E$ is not (essentially) a sheaf, then $E$ fits into an exact sequence in $\mathcal{B}_{0, H}$,

$$
0 \rightarrow F_{1}[1] \rightarrow E \rightarrow F_{2} \rightarrow 0,
$$

such that $F_{i}$ are slope stable sheaves with $c_{1}\left(F_{1}\right)=[H], c_{1}\left(F_{2}\right)=-[H]$. The $\left.\nu_{t[H]}\right]^{- \text {stability implies }}$

$$
\nu_{t[H]}\left(F_{1}[1]\right) \leq \nu_{t[H]}(E)=0 \leq \nu_{t[H]}\left(F_{2}\right),
$$

which shows $\operatorname{ch}_{2}\left(F_{i}\right) H>0$. Hence we can apply Corollary 1.3 for each $F_{i}$ (and noting Remark 2.7) to conclude that $\operatorname{ch}_{3}(E) \leq 5 / 6$. This is enough to show the inequality conjectured in [3, Conjecture 1.3.1]. We hope that the above argument is generalized to the case that $c_{1}(E)$ is higher than $2[H]$ as an induction argument.

Remark 2.10. The argument of Remark 2.9 shows that, in the case of $c_{1}(E)=$ $2[H]$, the conjecture in [3, Conjecture 1.3.1] is reduced to the case that $E$ is a sheaf, e.g. $E$ is a rank one torsion free sheaf. However in this case, we need a Castelnuovo type inequality, stronger than the known one, to show the desired inequality in [3, Conjecture 1.3.1]. 


\section{REFERENCES}

[1] D. Arcara and A. Bertram, Bridgeland-stable moduli spaces for K-trivial surfaces, J. Eur. Math. Soc. (JEMS) 15 (2013), no. 1, 1-38. MR2998828

[2] A. Bayer, A. Bertram E. Macri, and Y. Toda, Bridgeland stability conditions on 3-folds II: An application to Fujita's conjecture. Preprint, arXiv:1106.3430.

[3] A. Bayer, E. Macri, and Y. Toda, Bridgeland stability conditions on 3-folds I: BogomolovGieseker type inequalities, J. Algebraic Geom. 23 (2014), no. 1, 117-163. MR3121850

[4] F. A. Bogomolov, Holomorphic tensors and vector bundles on projective manifolds (Russian), Izv. Akad. Nauk SSSR Ser. Mat. 42 (1978), no. 6, 1227-1287, 1439. MR522939 (80j:14014)

[5] Tom Bridgeland, Stability conditions on K3 surfaces, Duke Math. J. 141 (2008), no. 2, 241-291, DOI 10.1215/S0012-7094-08-14122-5. MR2376815 (2009b:14030)

[6] D. Gieseker, On a theorem of Bogomolov on Chern classes of stable bundles, Amer. J. Math. 101 (1979), no. 1, 77-85, DOI 10.2307/2373939. MR527826 (80j:14015)

[7] Daniel Huybrechts and Manfred Lehn, The geometry of moduli spaces of sheaves, Aspects of Mathematics, E31, Friedr. Vieweg \& Sohn, Braunschweig, 1997. MR:1450870 (98g:14012)

[8] Adrian Langer, Moduli spaces of sheaves and principal G-bundles, Algebraic geometrySeattle 2005. Part 1, Proc. Sympos. Pure Math., vol. 80, Amer. Math. Soc., Providence, RI, 2009, pp. 273-308. MR2483939 (2010d:14010)

[9] Y. Toda, Bogomolov-Gieseker type inequality and counting invariants, J. Topol. 6 (2013), no. 1, 217-250. MR3029426

[10] Kōta Yoshioka, An action of a Lie algebra on the homology groups of moduli spaces of stable sheaves, Algebraic and arithmetic structures of moduli spaces (Sapporo 2007), Adv. Stud. Pure Math., vol. 58, Math. Soc. Japan, Tokyo, 2010, pp. 403-459. MR2676164(2011j:14019)

Kavli Institute for the Physics and Mathematics of the Universe, Todai Institute For Advanced Studies (TODiAs), University of Tokyo, 5-1-5 Kashiwanoha, Kashiwa, 277-8583, JAPAN

E-mail address: yukinobu.toda@ipmu.jp 